\title{
Individual aortic baroreceptors are sensitive to different ranges of blood pressures
}

\author{
CHEN MengJiao, YANG MingHao, HAN WenJuan, AN ShuCheng, LIU YiHui, \\ LIU ZhiQiang \& REN Wei*
}

MOE Key Laboratory for Modern Teaching Technology and College of Life Sciences, Shaanxi Normal University, Xi'an 710062, China

Received October 15, 2013; accepted March 5, 2014; published online April 15, 2014

\begin{abstract}
Many receptors, including thermal receptors and mechanical receptors, are only activated by stimuli within a clearly defined range of intensities. Differences in the receptive ranges enable individual receptors and their sensory centers to precisely detect the intensity of the stimulus and changes in intensity. Baroreceptors are the sensory terminals of the baroreflex. It is well understood that an increasing number of baroreceptors are recruited to produce afferent action potentials as the blood pressure increases, indicating that individual baroreceptors have different pressure thresholds. The present study revealed that individual baroreceptors could stop their afferent signals when the blood pressure exceeds a certain level, indicating that individual baroreceptors are sensitive to a specific range of blood pressure. The receptive ranges of individual baroreceptors differ in terms of the total range, the lower threshold, and the upper threshold. Of 85 baroreceptors examined in this study, the upper thresholds for about half were within the physiological blood pressure range. These results indicate that supraphysiological blood pressure is unlikely to be encoded by the recruitment of more baroreceptors. Instead, supraphysiological blood pressure levels might be signaled by an increase in the frequency of action potentials or by other mechanisms. In conclusion, our results indicate that rabbit baroreceptors are activated by blood pressure levels within specific receptive ranges. These findings should encourage further studies to examine the role of population coding of blood pressure by baroreceptors in the baroreflex.
\end{abstract}

depolarization block, receptive range, rabbit depressor nerve, single fiber recording

Citation: Chen MJ, Yang MH, Han WJ, An SC, Liu YH, Liu ZQ, Ren W. Individual aortic baroreceptors are sensitive to different ranges of blood pressures. Sci China Life Sci, 2014, 57: 502-509, doi: 10.1007/s11427-014-4649-7

The baroreflex is the major physiological mechanism involved in the rapid regulation of blood pressure. Stretching of the aorta following an increase in blood pressure activates baroreceptors located in the carotid sinus and aorta, causing stronger afferent signal to the blood pressure regulatory center. The reflex system lowers blood pressure by decreasing the heart rate and the peripheral resistance. This reflex is also known as the depressure reflex [1,2]. Baroreceptors, sensory nerve terminals located beneath the vascular adventitia, are the sensory components of the depressure reflex. Variations in blood pressure cause changes in vascu-

*Corresponding author (email: renwei@snnu.edu.cn) lar extension and thus the stress on baroreceptors. Afferent neural impulses generated by the baroreceptors then convey information regarding the changes in blood pressure to the central nervous system [3].

It is well established that, within the physiological range of blood pressure, the frequency of impulses generated by individual baroreceptors is positively correlated with vascular extension, with an S-shaped correlation between the frequency of afferent impulses and blood pressure [4-7]. As blood pressure increases, more baroreceptors are recruited and changes in blood pressure are coded by the frequency of action potentials derived from the individual afferent nerve fibers and the number of the recruited active fibers. These 
signaling patterns imply that individual baroreceptors have specific receptive ranges, because different fibers are activated at different blood pressure levels $[8,9]$.

For thermal and mechanical receptors, the receptive range is defined as the intensity range of the stimulus that induces afferent signals from the corresponding receptor. For example, some thermal receptors only fire at low temperatures and rest at higher temperature while others only fire at higher temperatures. Because different fibers in the thermal afferent nerve are activated by different temperatures, the thermal sensory center can detect different temperatures and initiate appropriate reflex responses. The individual thermal receptors start firing at their preset lower temperature threshold and stop firing once the temperature exceeds the higher threshold. Therefore, these thresholds denote the receptor's range. By acting in a cooperative manner, a population of thermal receptors, each with distinct thresholds, can detect and encode a range of external temperatures [10-12].

It is well known that individual baroreceptors start firing at different blood pressure levels. However, it is currently unknown whether they stop firing as the blood pressure increases. Therefore, do individual baroreceptors have limited receptive ranges, like thermal receptors, where firing starts at a lower threshold and stops at a higher threshold? This question needs to be systematically investigated. Although previous studies clearly show there is a pressure threshold for initiating firing, there is no clear conclusion on the pressure threshold for stopping firing. It is believed that the firing frequency of baroreceptors may increase to a maximum rate and become saturated as blood pressure continues to increase. However, there is no clear evidence for whether baroreceptors stop firing during further increases in blood pressure [13].

It was also reported that, even if blood pressure is in the physiological range, some baroreceptors generate a temporal pattern in which they stop firing (i.e., rest) in the systolic phase but resume firing in the diastolic phase $[14,15]$. The results indicate that at least some baroreceptors can stop firing as blood pressure increases in the systolic phase. These findings imply that some baroreceptors exhibit a limited receptive range, even at physiological blood pressure levels. To address these issues, the present study assessed the blood pressure levels corresponding to the start and end of firing of rabbit aortic baroreceptors. The results demonstrated the existence and general characteristics of a receptive range of rabbit baroreceptors. The results also highlight the need for future studies investigating the role of population coding by baroreceptors in the baroreflex.

\section{Materials and methods}

\subsection{Animals and electrophysiology}

Adult New Zealand rabbits weighing 2-2.5 kg were ob- tained from Doumen Experimental Animal Center (Xi'an, Shaanxi, China). The rabbits were cared for in strict accordance with the Guiding Principles for the Care and Use Committee of Shaanxi Normal University, and were housed two per cage in a fully accredited institution for $\geqslant 1$ week before use. The rabbits had free access to normal rabbit food and water. Before use, rabbits were anesthetized with urethane intravenously, at a dose of $1 \mathrm{~g} \mathrm{~kg}^{-1}$, and additional doses were given if necessary. The rabbits were also infused with prewarmed physiological saline via a catheter inserted in a femoral vein to avoid dehydration [16]. The body temperature of the rabbits was maintained at $37^{\circ} \mathrm{C}$ with a heating pad.

We used two complementary strategies to investigate the responses of baroreceptors to changes in blood pressure. In the first strategy, we observed the firing activity of baroreceptors induced by dynamic changes in blood pressure in vivo. This blood pressure change is dynamic and within the physiological range. In the second strategy, to investigate the effects of vascular blood pressure on firing activity, we artificially controlled the inner vascular pressure by isolating a segment of the rabbit aorta in situ [17].

\subsubsection{In vivo surgical procedures to expose the aorta and depressor nerve}

The rabbits were tracheotomized through a midline cervical incision and ventilated with room air mixed with oxygen. Polyethylene catheters were inserted into the right common carotid artery to measure arterial pressure and in the right femoral vein for drug administration. Aortic pressure was measured using a high-fidelity pressure transducer (ML221; ADInstruments, Sydney, Australia). To record aortic depressor nerve activity, the right aortic depressor nerve was carefully dissected and marinated in an oil pool. A thin bundle of depressor nerve fibers was separated, and its afferent firing trains were recorded using a fine platinum electrode with a nearby reference electrode connected to a bioelectrical amplifier. Blood pressure and spike trains of individual fibers were simultaneously recorded using a Powerlab system (ADInstruments) with a sampling frequency of $10.0 \mathrm{kHz}$. The system was observed throughout the study to ensure that each recording was of a single unit. The systemic blood pressure level was altered by femoral intravenous administration of norepinephrine (NE, 1:5000) or acetylcholine (ACh, 1:10000) after a stable baseline of $\geqslant 20 \mathrm{~min}$. The recovery processes after infusing NE or ACh were also observed.

\subsubsection{In situ surgical procedure to isolate the aorta and expose the depressor nerve}

A midline incision was made in the neck and upper chest. The sternum was cut and the upper chest was opened. The trachea was cannulated and ventilated. The innominate artery was ligated at its root. A polyethylene tube was inserted into the right common carotid artery at its middle portion. 
The tip of the polyethylene tubing was advanced to the bifurcation of the innominate artery. A fluid-filled pressure transducer and a servo-controlled pump system (LSP02-1B1; LongerPump, Baoding, China) were connected to the polyethylene tube. The right subclavian artery was ligated immediately proximal to its first branch at the vertebral or internal thoracic artery. In this way, we prepared a completely watertight chamber in the bifurcation of the innominate artery. The right aortic depressor nerve was identified and cut at its junction with the superior laryngeal nerve. Then, the nerve was desheathed. To directly measure the differences in the receptive ranges of individual baroreceptors, two single fibers were isolated and placed on individual platinum electrodes. The pressure and afferent spike trains of these two single fibers were simultaneously recorded. To determine the receptive range of individual baroreceptors, single nerve fibers were isolated and their afferent potentials were recorded. The recording site was immersed in a pool of mineral oil. After stabilization for 5 min during which time the vascular pressure in the isolated region was kept at 80 $\mathrm{mmHg}$, the pressure was gradually increased from 30 to 300 $\mathrm{mmHg}$ at a rate of $<2 \mathrm{mmHg} \mathrm{s}^{-1}$ [18] using the servocontrolled pump.

\subsection{Data analysis}

When the nerve fiber stopped firing following an increase in blood pressure induced by $\mathrm{NE}$ in vivo, the mean blood pressure in one cardiac cycle at the last burst of firing was calculated as the upper pressure threshold. After injecting $\mathrm{ACh}$, the mean blood pressure in one cardiac cycle at the last observed burst was calculated as the lower pressure threshold.

When the vascular pressure in the isolated aortic segment was increased gradually from 30 to $300 \mathrm{mmHg}$, the mean blood pressure corresponding to the first five action potentials was taken as the lower pressure threshold while the mean pressure corresponding to the last five action potentials was taken as the upper pressure threshold.

The time between the maximum values of successive spikes was recorded as the interspike interval (ISI). Numerical data are expressed as the mean \pm standard deviation (SD).

\section{Results}

\subsection{In vivo recording of changes in firing of single de- pressor nerve fibers caused by changes in blood pressure}

We typically observed bursts of firing when blood pressure changes and the firing of single depressive nerve fibers were simultaneously recorded in rabbits in vivo. These bursts occurred in the systolic phase and gaps between two successive bursts were apparent in the diastolic phase. During transient NE-induced increases in arterial pressure (Fig- ure 1A), the firing pattern switched from bursts (Figure 1B) to continuous firing (Figure $1 \mathrm{C}$ ), but then resumed a bursting pattern in the diastolic phase (Figure 1D). The number of spikes in each burst gradually decreased to the final resting state (Figure 1E). As the blood pressure recovered to the baseline level (i.e., as measured before NE injection), the resting fibers that generated spikes in the diastolic phase displayed a bursting pattern if the bursts appeared in the diastolic period (Figure 1F). The burst pattern was restored if the bursts initially appeared in the systolic phase (Figure $1 \mathrm{H}$ ) following a continuous firing pattern (Figure $1 \mathrm{G}$ ).

Twenty minutes after the blood pressure had returned to the baseline level, ACh was injected to transiently reduce blood pressure. During this reduction of blood pressure, the bursting firing pattern of the single depressor nerve fiber gradually declined in terms of the number of spikes per burst (Figure 1A). The bursting firing pattern observed at baseline blood pressure resumed as the blood pressure recovered.

The injections of NE and Ach caused blood pressure to increase and recover, and then to decrease and recover, respectively. In this process, the single depressive nerve fiber stopped firing at a blood pressure level corresponding to the upper threshold of the receptive range of its baroreceptor. The fiber also stopped firing at a blood pressure level corresponding to the lower threshold of the receptive range of its baroreceptor. Using this experimental method, we were able to measure the receptive range of the baroreceptor connected to this recorded single depressor nerve fiber. The receptive range was limited and defined by a lower pressure threshold at which firing started and an upper threshold at which firing stopped.

Using this experimental method changing the mean blood pressure from 65 to $160 \mathrm{mmHg}$, similar changes in firing patterns were observed for 17 single depressor nerve fibers. Eight of these fibers stopped firing during increases and decreases in blood pressure, displaying clear lower and upper pressure thresholds. If the changes in blood pressure during each cardiac cycle cross the lower or upper thresholds, the baroreceptors will dynamically switch between resting and firing, resulting in a bursting firing pattern. During large, slow increases in blood pressure, a regular firing cycle becomes apparent, consisting of periods of rest, systolic bursts (i.e., bursts in the systolic phase), continuous firing (i.e., the spiking frequency oscillates in each cardiac cycle), diastolic bursts (i.e., bursts in the diastolic phase), and back to rest. The systolic burst is generated by dynamic and repetitive crossing of the blood pressure level across the lower threshold of the receptive range in vivo, while the diastolic burst is generated by dynamic and repetitive crossing of blood pressure across the upper threshold.

For nine single fibers, the firing pattern differed slightly from the scenario described above. Intriguingly, these nine fibers did not exhibit both lower and upper thresholds, 

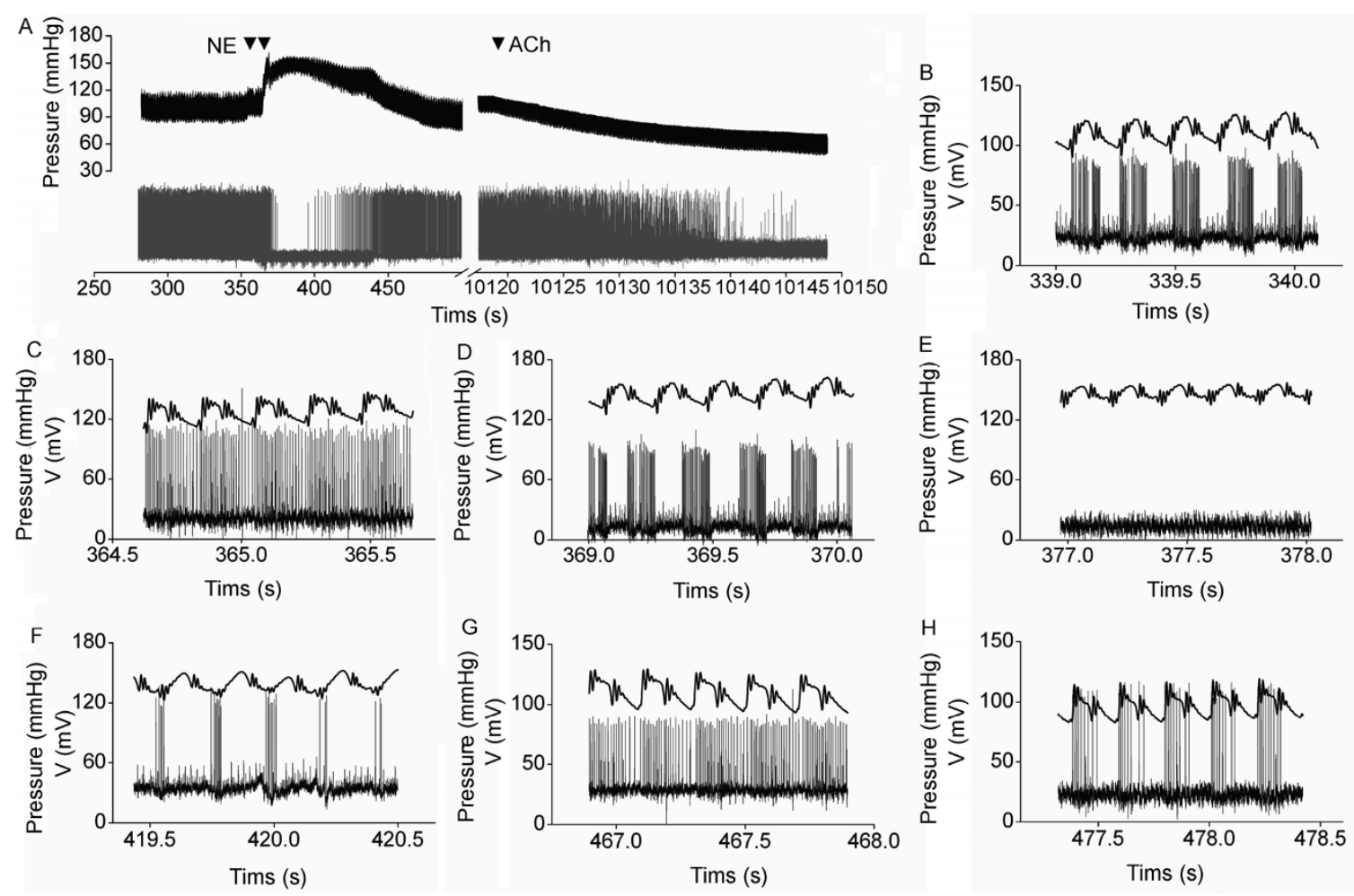

Figure 1 Firing patterns of a typical aortic baroreceptor induced by changes in blood pressure in vivo. A, Changes in firing patterns induced by NE (1:5000; $\boldsymbol{\nabla})$ and $\mathrm{ACh}(1: 10000, \boldsymbol{\nabla})$. B, This baroreceptor displayed a systolic bursting pattern at physiological blood pressures. Injection of NE induced the baroreceptor to burst in a continuous manner $(\mathrm{C})$, followed by diastolic bursts (D) and rest (E). As blood pressure returned to the baseline level, the baroreceptor resumed diastolic bursting $(F)$, continuous firing $(G)$, and systolic bursting $(H)$.

which indicates that the receptive ranges of the baroreceptors connected to those nine fibers did not cover the range of blood pressures induced by NE or ACh in this experimental setting. Therefore, we used an in situ isolation protocol to artificially control the vascular pressure of the rabbit aorta across a wider range and therefore detect the receptive ranges of the upstream baroreceptors.

\subsection{Simultaneous recording of two single fibers during changes in blood pressure}

To detect possible differences in the receptive ranges of individual baroreceptors, we simultaneously recorded the changes in firing activity of two single depressor nerve fibers while gradually increasing the vascular pressure of a segment of the isolated aorta. As shown in Figure 2, both fibers exhibited regular changes in their firing patterns, including initiation of firing, continuous firing at an increasing frequency, and stopping of firing. Notably, the lower and upper pressure thresholds at the start and end of firing differed between the two fibers, which indicates that the baroreceptors connected to the two fibers had different receptive ranges. The results provide strong evidence that the receptive ranges of individual aortic baroreceptors are limited and differ markedly between receptors ( $n=3$ pairs).

\subsection{Pressure thresholds for the start and end of firing in 85 single depressor nerve fibers}

To assess the overall distribution of the receptive ranges of rabbit aorta baroreceptors, we recorded the activities of single depressor nerve fibers during gradually increasing the vascular pressure from 30 to $300 \mathrm{mmHg}$ using in situ preparations. We successfully recorded the activities of the right depressor nerves in 85 single fibers in a total of 24 rabbits. At this range of blood pressures, we observed lower and upper thresholds for 39 fibers. A further 40 fibers were already firing at a vascular pressure of $30 \mathrm{mmHg}$, indicating that their lower thresholds are $<30 \mathrm{mmHg}$. For statistical analyses, the lower threshold of these fibers was defined as $30 \mathrm{mmHg}$. The other 16 fibers were still firing when the vascular pressure reached $300 \mathrm{mmHg}$, indicating that their upper thresholds are $>300 \mathrm{mmHg}$. The upper threshold for these fibers was defined as $300 \mathrm{mmHg}$ in the statistical analyses.

The pressure thresholds at the start and end of firing in the 85 single depressor nerves reflect, respectively, the lower and upper thresholds of their corresponding baroreceptors. As shown in Figure 3, the receptive ranges differed markedly. The lowest and the highest values for the lower threshold were 30 and $170.74 \mathrm{mmHg}$, respectively (mean \pm SD: $56.38 \pm 34.63 \mathrm{mmHg}$ ) (Figure 3B), while lowest and 


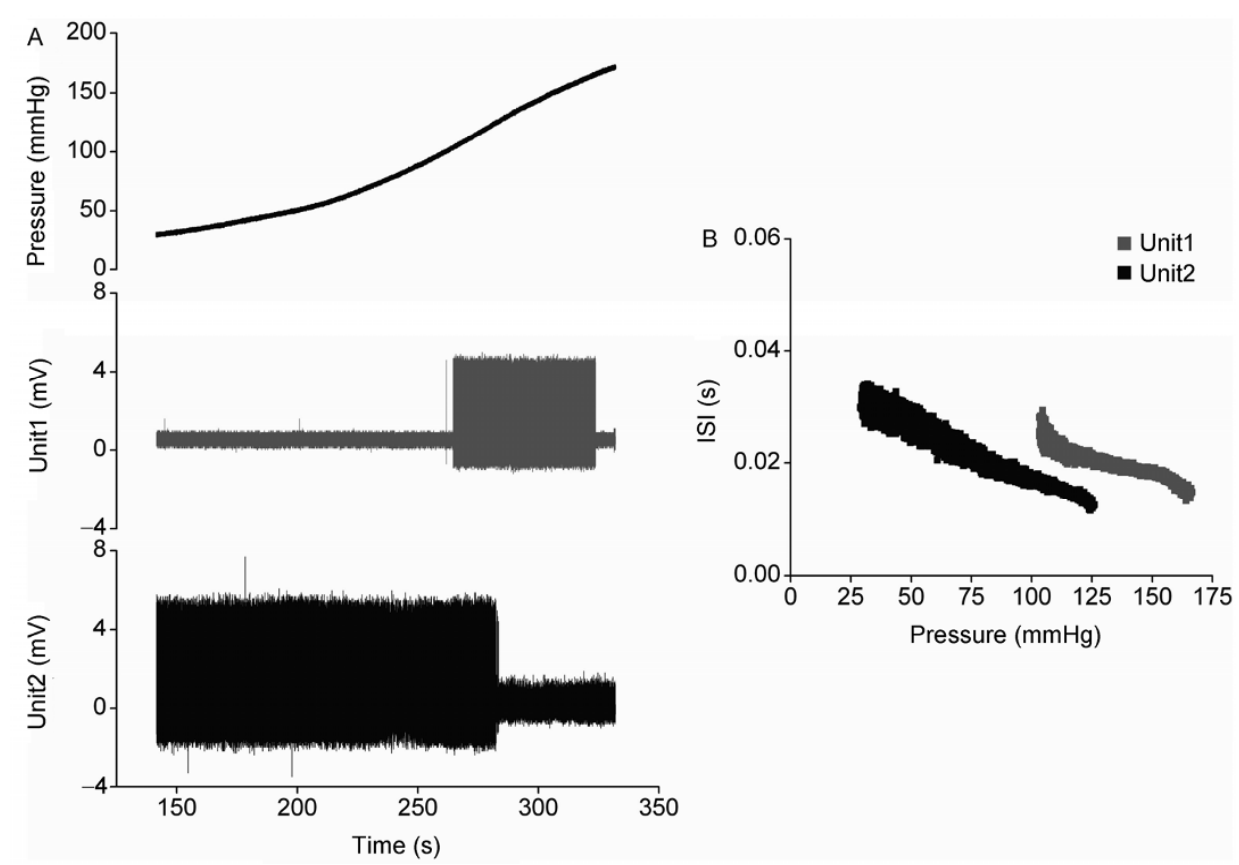

Figure 2 Simultaneous recording of the changes in firing patterns in response to an increase in vascular pressure in two depressor nerve single fibers (units). A, Changes in vascular pressure and firing activities of the two units. In unit one, firing started at $102 \mathrm{mmHg}$ and stopped at $167 \mathrm{mmHg}$. In unit two, firing was apparent at $30 \mathrm{mmHg}$ and stopped at $127 \mathrm{mmHg}$. B. ISI-pressure curves demonstrate the distinct receptive ranges of both fibers, and confirm that ISI decreases (i.e., the firing frequency increases) as vascular pressure increases.

highest values for the upper threshold were 58.18 and 300 $\mathrm{mmHg}$, respectively (mean $\pm \mathrm{SD}: 219.26 \pm 67.81 \mathrm{mmHg}$ ) (Figure $3 \mathrm{C}$ ). Therefore, the absolute range of blood pressure values that could be detected by individual baroreceptor units varied from 26.7 to $270 \mathrm{mmHg}$ (mean $\pm \mathrm{SD}$ for the absolute range: $162.88 \pm 70.32 \mathrm{mmHg}$ ) (Figure $3 \mathrm{~A}$ ).

The number of active baroreceptors recruited at specific vascular pressures could be determined using the data shown in Figure 3A. As shown in Figure 3D, only some of the 85 baroreceptors were active at specific vascular pressures. The number of recruited fibers reached a maximum at $125 \mathrm{mmHg}$ but declined at higher vascular pressures. These results indicate that the number of recruited baroreceptors and the frequency of firing from each baroreceptor can depict changes in blood pressure at levels $<125 \mathrm{mmHg}$. However, if the blood pressure is $>125 \mathrm{mmHg}$, the number of active baroreceptors decreases as blood pressure increases. Therefore, above this level, the increase in blood pressure is no longer represented by the recruitment of additional baroreceptors, but might be encoded by increases in the firing frequency in the active baroreceptors or by other methods.

\subsection{Complex firing rhythms of single depressor nerve fibers near the thresholds for starting or stopping firing}

Previous neurodynamic studies demonstrated that if the membrane potential of a neuron is held very near to its excitation threshold, it will exhibit spontaneous and specific firing patterns. The dynamic properties of the complex fir- ing patterns of neurons highlight the dynamic bifurcation mechanisms underpinning the generation of firing patterns when a neuron is at the threshold of excitation [19,20]. Consistent with this concept, we observed complex firing patterns of single nerve fibers when the vascular pressure of an isolated aorta segment was carefully adjusted to a pressure very close to the fiber's lower or upper threshold. A typical example of this phenomenon is shown in Figure 4A. When the vascular pressure was increased across the lower threshold, the baroreceptor started to fire intermittently, alternating between a firing train and long periods of rest (Figure 4B1). This firing pattern was defined as an intermittent pattern. The ISI of the firing train showed relatively wide variation. The first return map of the ISI is shown in Figure 4C1, which indicates that subcritical Hopf bifurcation dynamics underpin this lower threshold of excitation. Following this point, the firing became continuous (Figure 4B2) and the ISI decreased with increasing vascular pressure. The first return map of periodic firing is shown in Figure 4C2. When the vascular pressure was increased to a level that held the baroreceptors near the threshold for depolarization block, the firing rhythm exhibited a characteristically layered structure in its ISI (Figure 4A). The first return map of this ISI is presented by a lattice structure (Figure 4C3). The firing pattern of this feature is defined as a multiple integer rhythm, indicating that supercritical Hopf bifurcation dynamics underpin the threshold for depolarization block.

The present study revealed that, at the lower pressure 

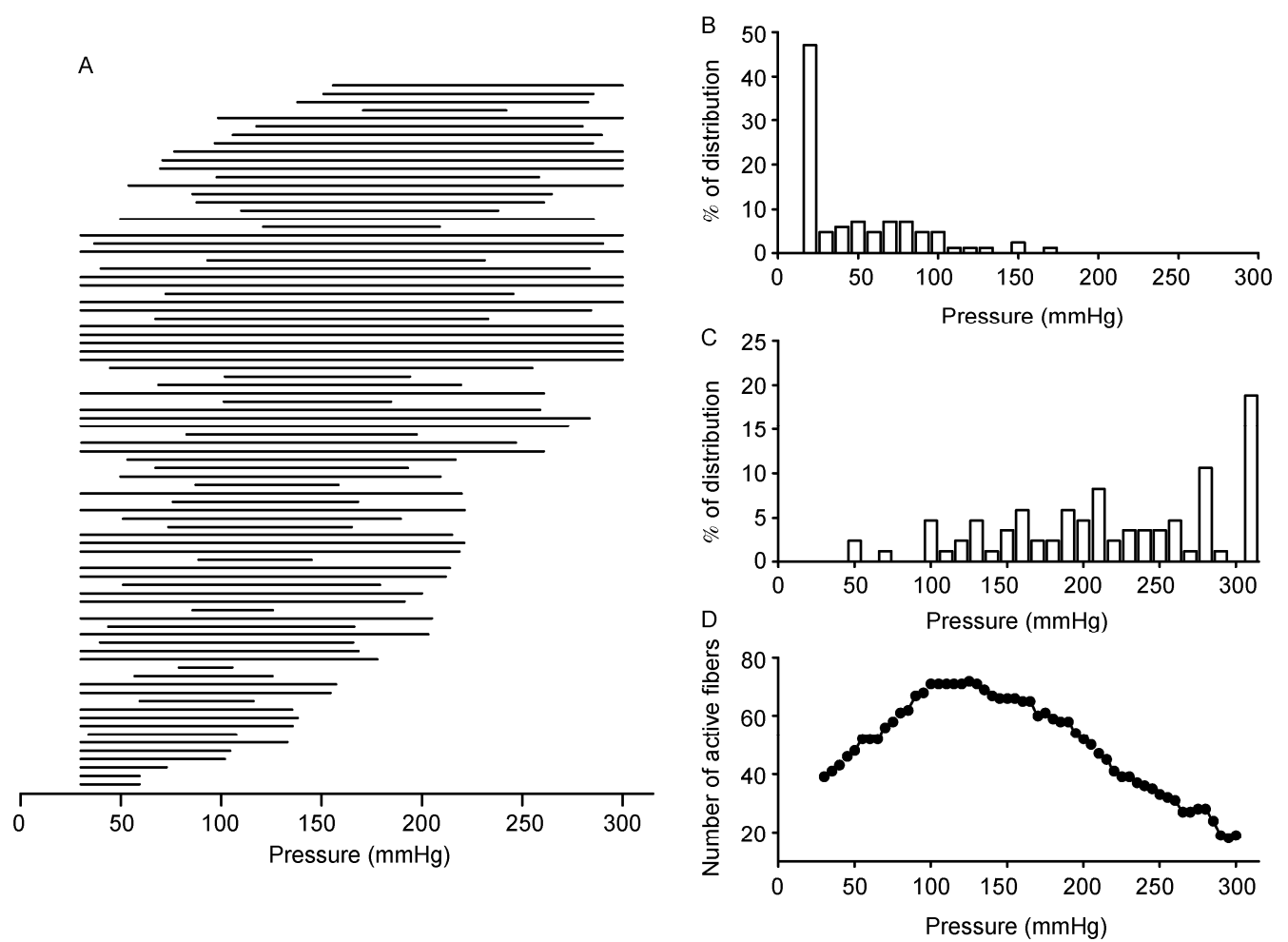

Figure 3 Receptive ranges of 85 rabbit aortic baroreceptors. A, Pressure range covered by the receptive ranges of the individual baroreceptors. B, Distribution of the lower pressure thresholds. C, Distribution of the upper pressure thresholds. D, Number of active baroreceptors recruited at specific vascular pressures.

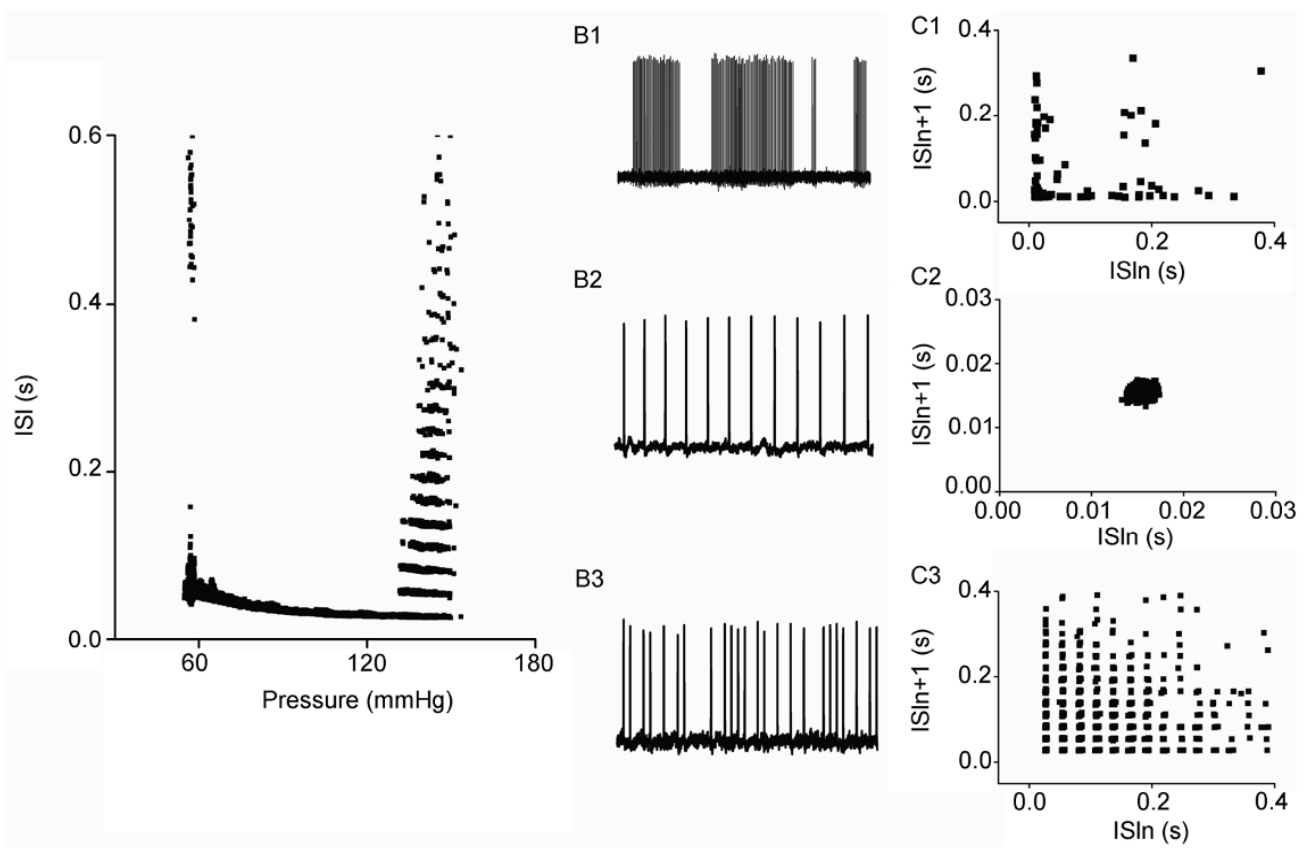

Figure 4 Effects of increasing vascular pressure on the changes in firing patterns in isolated aorta segments in situ. A, Overall changes in firing activities. $\mathrm{B}$ and $\mathrm{C}$, An increase in vascular pressure led to sustained firing and intermittent periods of rest (B1) at $57 \mathrm{mmHg}$, characteristic of the first return map of ISI (C1). A stable period one firing (B2) and the first return map of ISI (C2). At $135 \mathrm{mmHg}$, integer multiple firing appeared (B3), exhibiting lattice structure in its first return map (C3) corresponding to a layered ISI (A).

threshold, 12 baroreceptors started to fire from rest with a multiple integer firing pattern, while 29 baroreceptors fired with an intermittent firing pattern. At the upper threshold, 49 baroreceptors stopped firing through the multiple integer 
firing pattern, while nine baroreceptors stopped firing at an intermittent firing pattern.

Analysis of the dynamics of the firing rhythms close to the threshold levels revealed that neural excitability was responsible for the lower threshold of the baroreceptors while depolarization block was responsible for the upper threshold. When the vascular pressure increases to the lower pressure threshold of the receptive range, the receptor potential drives the membrane potential of the spike-initial segment to move to the threshold of excitability, resulting in spike generation and the formation of a firing train. When the vascular pressure increases to the upper pressure threshold of the receptive range, the receptor potential causes the membrane potential of the spike-initial segment to cross the threshold for depolarization block, resulting in overdepolarization of the initial segment and cessation of firing.

\section{Discussion}

Populations of receptors that sense the intensity of physical or chemical stimuli can encode a range of intensities by recruiting individual receptors with different receptive ranges. In such populations, individual receptors start firing at a low stimulus intensity and stop firing at a higher stimulus intensity, and each receptor has a limited and distinct receptive range. Differences in the receptive ranges of individual receptors are necessary for the receptor population to detect different levels of stimulus intensity. In the present study, we first found that the firing of rabbit aortic baroreceptors started and stopped in response to changes in blood pressure levels within the physiological range. We recorded a series of regular firing patterns when the blood pressure was varied within physiological ranges to activate the baroreceptors. By artificially controlling the vascular pressure of a segment of isolated aorta in situ, we found that pairs of single nerve fibers displayed distinct blood pressure thresholds even though vascular pressure was identical for both fibers. By observing 85 individual baroreceptors, we found that individual aortic baroreceptors in rabbit exhibit limited and distinct receptive ranges. Notably, there were marked differences in the pressure range covered and the upper and lower thresholds of the receptive ranges among individual nerve fibers. We also found that the upper pressure threshold that stopped the firing of many baroreceptors was within the physiological blood pressure range. Therefore, there was no increase or even decrease in the number of baroreceptors that were recruited when blood pressure was increased to a supraphysiological level. These results indicate that at supraphysiological blood pressure, the change in blood pressure is no longer represented by the recruitment of active fibers in the depressor nerve, and might be depicted by an increase in the firing frequency or another method instead.

Aortic baroreceptors are stretch-sensitive mechanical receptors. An increase in blood pressure extends the blood vessel and its receptors, increasing the membrane potential and causing depolarization of the spike-initial segment to the excitation threshold, representing an all-or-none action potential. Therefore, the lower pressure threshold of individual baroreceptors is determined by this mechanism of excitation and the pressure value corresponding to the excitability threshold is the lower limit of the receptive range. As observed in the present study, rabbit aortic baroreceptors also stopped firing despite further increases in blood pressure above a threshold level. This mechanism is thought to involve depolarization block. Direct intracellular recording of the receptor may reveal over-depolarization and the cessation of spike generation, and could confirm our assumption. However, it is very difficult to perform intracellular recording of the baroreceptor and the initial axon segment simultaneously, because both of these components are tiny and are embedded in the blood vessel. Therefore, we observed the stable firing rhythms that were spontaneously generated near the threshold level from firing to rest during over-depolarization. The observed multiple integer rhythm and the intermittent rhythm indicate that depolarization block of rabbit aortic baroreceptors is mediated by Hopf bifurcation, a common and dynamic mechanism of excitability.

The results of the present study might be of general physiological importance. Previous studies have shown that different fibers in the depressor nerve innervate different regions of the blood pressure regulation center. Considering the anatomical specificity of individual fibers and the variations in the receptive ranges of individual fibers of the depressor nerve, we think it is vital to investigate whether the anatomical specificity is related to the receptive range of fibers in depressor nerve. Depressor nerve fibers are mainly of types A and C. Type A fibers are fast conductors and start firing at lower pressure levels while type $\mathrm{C}$ fibers are slow conductors and start firing at relatively higher pressure levels [8]. These two types of fibers innervate different subregions of the nuclei tractus solitarii, and they rarely terminate on the same neurons [21]. It is interesting to note that neurons in the nuclei tractus solitarii can be categorized according to their firing patterns into two subtypes; one subtype starts firing at lower pressure levels while the other starts firing at higher pressure levels [22]. Neurons firing at lower pressure thresholds are mainly innervated by type A fibers, which also start firing at lower pressure thresholds. By contrast, neurons firing at higher thresholds are mainly innervated by type $\mathrm{C}$ fibers, which also start firing at higher thresholds [23]. These results indicate that the afferent projections of different types of baroreceptors are different, and these differences might be important in the stimulus encoding by the population of baroreceptors.

In conclusion, the results of our study indicate that baroreceptors, like thermal receptors and stretch receptors, start signaling at a low stimulus threshold and stop signaling at a high stimulus threshold, thus manifesting a receptive range. 
Afferent fibers with different receptive ranges may project to central neurons located in different subregions of the nuclei tractus solitarii, where they activate different reflex responses, allowing delicate control of the depressure reflex. Our novel findings mean it is necessary to modify the traditional view of how changes in blood pressure variation are encoded by the baroreceptor population. Our results should also introduce new directions for future research examining the afferent signaling of blood pressure changes and the delicate control of the depressure reflex.

This work was supported by the National Natural Science Foundation of China (30770701) and the Fundamental Research Funds for the Central Universities (GK201302052, GK261001007).

1 Kirchheim HR. Systemic arterial baroreceptor reflexes. Physiol Rev, 1976, 56: 100-177

2 Sunagawa K, Sato T, Kawada T. Integrative sympathetic baroreflex regulation of arterial pressure. Ann N Y Acad Sci, 2001, 940: 314-323

3 Grigg P. Biophysical studies of mechanoreceptors. J Appl Physiol, 1986, 60: 1107-1115

4 Heymans C. Reflexogenic areas of the cardiovascular system. Perspect Biol Med, 1960, 3: 409-417

5 McCubbin JW, Green JH, Page IH. Baroceptor function in chronic renal hypertension. Circ Res, 1956, 4: 205-210

6 Krieger EM, Marseillan RF. Aortic Depressor Fibers in the Rat: An Electrophysiological Study. Am J Physiol, 1963, 205: 771-774

7 Pelletier CL, Clement DL, Shepherd JT. Comparison of afferent activity of canine aortic and sinus nerves. Circ Res, 1972, 31: 557-568

8 Seagard JL, van Brederode JF, Dean C, Hopp FA, Gallenberg LA, Kampine JP. Firing characteristics of single-fiber carotid sinus baroreceptors. Circ Res, 1990, 66: 1499-1509

9 van Brederode JF, Seagard JL, Dean C, Hopp FA, Kampine JP. Experimental and modeling study of the excitability of carotid sinus baroreceptors. Circ Res, 1990, 66: 1510-1525

10 McKemy DD. Temperature sensing across species. Pflugers Arch, 2007, 454: 777-791
11 Chatzigeorgiou M, Yoo S, Watson JD, Lee WH, Spencer WC, Kindt KS, Hwang SW, Miller DM, 3rd, Treinin M, Driscoll M, Schafer WR Specific roles for DEG/ENaC and TRP channels in touch and thermosensation in C. elegans nociceptors. Nat Neurosci, 2010, 13: 861-868

12 Patapoutian A, Peier AM, Story GM, Viswanath V. ThermoTRP channels and beyond: mechanisms of temperature sensation. Nat Rev Neurosci, 2003, 4: 529-539

13 Saum WR, Ayachi S, Brown AM. Actions of sodium and potassium ions on baroreceptors of normotensive and spontaneously hypertensive rats. Circ Res, 1977, 41: 768-774

14 Yang MH, An SC, Gu HG, Liu ZQ, Ren W. Understanding of physiological neural firing patterns through dynamical bifurcation machineries. Neuroreport, 2006, 17: 995-999

15 Yang MH, An SC, Gu HG, Liu ZQ, Ren W. How blood pressure is encoded in firing pattern in aortic arch baroreceptor? In: Proceedings of the Second International Conference on Dynamics Vibration and Control, Beijing, China, 2006. 116-117

16 DiBona GF, Sawin LL. Reflex regulation of renal nerve activity in cardiac failure. Am J Physiol, 1994, 266: R27-39

17 Sato T, Kawada T, Miyano H, Shishido T, Inagaki M, Yoshimura R, Tatewaki T, Sugimachi M, Alexander J Jr., Sunagawa K. New simple methods for isolating baroreceptor regions of carotid sinus and aortic depressor nerves in rats. Am J Physiol, 1999, 276: H326-332

18 Andresen MC, Yang M. Gadolinium and mechanotransduction of rat aortic baroreceptors. Am J Physiol, 1992, 262: H1415-1421

19 Gu HG, Ren W, Yang MH, Lu QS. A Novel bursting in the experimental neuronal pacemaker, intermittent period 1 spiking (in Chinese). Acta Biophys Sin, 2002, 18: 440-447

20 Gu HG, Ren W, Lu QS, Yang MH. The bifurcation and integer multiple spiking in the experimental neuronal pacemakers (in Chinese). Acta Biophys Sin, 2001, 17: 637-644

21 Donoghue S, Fox RE, Kidd C, McWilliam PN. The terminations and secondary projections of myelinated and non-myelinated fibres of the aortic nerve in the cat. Q J Exp Physiol, 1981, 66: 405-422

22 Seagard JL, Dean C, Hopp FA. Discharge patterns of baroreceptor-modulated neurons in the nucleus tractus solitarius. Neurosci Lett, 1995, 191: 13-18

23 Bailey TW, Jin YH, Doyle MW, Andresen MC. Vanilloid-sensitive afferents activate neurons with prominent A-type potassium currents in nucleus tractus solitarius. J Neurosci, 2002, 22: 8230-8237

Open Access This article is distributed under the terms of the Creative Commons Attribution License which permits any use, distribution, and reproduction in any medium, provided the original author(s) and source are credited. 